\title{
Role of DNA methylation as a diagnostic biomarker of sporadic breast cancer
}

\author{
Wirsma Arif Harahap* \\ Medical School of Andalas University \\ DOI: http://dx.doi.org/10.19106/JMedScieSup004804201636
}

\section{ABSTRACT}

The initiation and progression of breast cancer have been recognized to be secondary to the accumulation of genetic mutations, which eventually lead to aberrant cellular function. Both inherited and sporadic genetic mutations might result in the activation of oncogenes and inactivation of tumor suppressor genes. Recent discovery revealed that reversible alterations in histone proteins and deoxyribonucleic acid (DNA) might also lead to tumorigenesis, thus introducing a novel term to the field of cancer research: epigenetics. Epigenetics refers to the study of heritable changes in gene regulation that do not involve a change in the DNA sequence. The most frequently discussed topic in epigenetics of breast cancer is promoter DNA methylation. DNA methylation is one of the hallmarks of cancer and plays a crucial role in oncogenesis, i.e. transcription blockade, supporting the notion that cellular inhibition might take place. Compared to normal tissues, double to triple hypermethylation occurs in cancerous cells.-

Detection of aberrantly-methylated $\mathrm{CpG}$ islands in promoter region of several genes in DNA sample derived from nipple aspirates, serum, or cancer tissue is associated with downregulation of gene expression and loss of function that would then lead to early stages of breast cancer with poorer prognosis. DNA methylation has been identified as signature for TNBC. Methylation of BRCA1 gene is frequently demonstrated in young, estrogen receptor-negative breast cancer patients. BRCA1 methylation in premenopausal women with sporadic breast cancer in West Sumatra region has been higher than in Western women, thus signifying the difference of methylation profile across race and socioeconomic status.

DNA methylation might be used in enhancement of current breast cancer classification. There is such distinction between methylation and gene expression profiles of breast cancers; that not all methylation profiles fit within the same molecular subtype. Specific gene methylation profiles are identified as basallike, luminal A and HER2-overexpressing breast cancers. A number of studies have analyzed the methylation status of BRCA1, a key player in TNBC. One study demonstrated that $B R C A 1$ promoter was methylated in TNBC, proven by the increasing sensitivity of TNBC cell lines to PARP inhibitors. In addition,

$\overline{\text { Corresponding author: wirsma_arif@yahoo.com }}$ 
BRCA1 methylation quantity was higher in patients with complete response to neoadjuvant chemotherapy than in non-responders.

Epigenetics is now the cutting edge of cancer research. Advances in this field would have major implications in diagnosis, prevention, treatment of cancer, and formulation of new epigenetically-targeted anticancer drugs.

Keywords: breast cancer, epigenetic, DNA methylation, BRCA1, diagnostic marker 